\title{
A High-Volume, Academic Orthopedic Specialty Hospital Provides the Shortest Operative Times in Total Hip Arthroplasty
}

\author{
Stephen G. Zak, BA ${ }^{1}$ Jenna Bernstein, MD ${ }^{1}$ Alex Tang, BS ${ }^{1}$ James Slover, MD ${ }^{1}$ \\ Ran Schwarzkopf, MD, MSc ${ }^{1}$ Joshua Rozell, MD ${ }^{1}$
}

${ }^{1}$ Department of Orthopedic Surgery, NYU Langone Health, New York City, New York

Address for correspondence Joshua Rozell, MD, Department of Orthopedic Surgery, NYU Langone Health, E 17th Street New York

J Hip Surg 2021;5:127-134. City, NY 10003 (e-mail: joshua.rozell@nyulangone.org).

\begin{abstract}
\section{Keywords}

- operative time

- total hip arthroplasty

- value-based care

- surgical volume

- academic hospital

- community hospital

Operative time is a potential modifiable target for reducing cost and optimizing efficiency in total hip arthroplasty (THA). Shorter operative times are associated with decreased costs and complications. One proposed solution for reducing operating room (OR) times is to preferentially perform THAs at a higher volume center. The purpose of this study is to investigate the impact of different hospital settings on mean operative time in THA. A retrospective analysis of 7,694 consecutive primary and revision THA cases across four different hospitals was conducted. The hospitals reviewed in this study include a high-volume academic (HVA) orthopedic hospital, a large tertiary, low-volume academic (LVA) center, a high-volume community (HVC) hospital, and a low-volume community (LVC) hospital. Operative times, patient baseline demographics, and procedure classification (inpatient vs. outpatient) were collected. For primary THA, the HVA hospital maintained the statistically lowest total OR times followed by the HVC hospital ( 155.96 vs. 167.27 minutes, $p<0.0001$ ). The LVC and HVC centers had similar total OR times (175.48 vs. 167.27 minutes, $p=0.578$ ). For revision THA, the LVC center had the shortest total OR time followed by the HVA, LVA, and HVC sites (194.85 vs. 224.69 vs. 244.07 vs. 279.54 minutes). However, there were a larger amount of revision cases performed at our HVA sites than our community hospitals (523 vs. 123 cases). Our HVA hospital specialized in orthopedic care experiences shorter primary THA OR times than community hospitals. These findings suggest that in addition to hospital volume, factors such as specialized staffing, standardized OR protocols, and additional resources affect operative times, making HVA centers better equipped to handle primary THA.
\end{abstract}

In an era of quality and value-based care in total joint arthroplasty (TJA), an important area of research has focused on targeting modifiable and nonmodifiable factors to optimize outcomes and improve efficiency of care. ${ }^{1-5}$ A potential modifiable target to optimize TJA cost and value is the length of operative time. Shorter operative time not only leads to

received

November 16, 2020

accepted after revision

May 10, 2021

published online

July 28, 2021

decreased operating room (OR) and anesthesia costs, but has also been shown in multiple studies to be associated with decreased complication rates, including deep infections, skin and soft tissue infections, readmissions, wound complications, reoperation rates, transfusion rates, rates of venousthromboembolism, and risk for neurologic complication. ${ }^{6-14}$

(c) 2021. Thieme. All rights reserved. Thieme Medical Publishers, Inc., 333 Seventh Avenue, 18th Floor, New York, NY 10001, USA
DOI https://doi.org/ 10.1055/s-0041-1731715. ISSN 2472-8446. 
This in turn has implications in the 90-day postacute care period and bundled payment programs.

Several studies have investigated the role of performing TJA in different hospital settings and the impact on mean operative times. One proposed solution for improving the value and efficiency of care is to preferentially perform total hip arthroplasties (THA) and total knee arthroplasties (TKA) at higher volume centers. In a recent study by Khanuja et al, the authors found that the community hospitals in their hospital system had significantly faster operating room time compared with tertiary academic hospitals. ${ }^{15}$ Contrary to previous findings, their study did not find that surgeon volume had any impact on surgical time. ${ }^{16-18}$ However, a main limitation of their study is that in their hospital system, the community hospital functioned as a pseudo-specialty hospital potentially increasing its efficiency in comparison to the academic hospitals. Moreover, these studies do not discuss revision surgeries. Thus, there is still some uncertainty regarding the efficacy of time-saving measures in TJA among varied hospital settings.

In our study, we sought to investigate whether these findings were translatable to primary and revision THA in our own institution. Our institution consists of a large, tertiary academic center, a high-volume academic (HVA) orthopedic hospital, and two lower-volume community hospitals. The primary objective of our study was to compare the differences in total OR times between all of the hospitals within our health system. Additionally, we evaluated various phases of OR time to be able to identify more specifically where the differences exist between each of the hospital settings. We hypothesized that our academic specialty hospital would have the shortest OR times as a result of the most specialized staff, accessibility to implants and equipment, and resources allowing for the greatest efficiency.

\section{Patients and Methods}

A retrospective review was conducted across four medical centers that operate under a single umbrella hospital system. The total number of consecutive patients who underwent a primary or revision THA between January 2016 and September 2019 was identified by using the procedure name listed on the operative logs. Conversion THA was excluded from this study because they are known to have exceptionally long operative times due to the complex nature of the cases which would skew the results. Operative times including total OR time, wheels-in to prep start, prep time (which includes patient positioning, sterile skin prep, and draping), prep-end to incision, incision time, and incision close to wheels-out (of the OR) were collected for each of the cases from institutional electronic medical records (EMR) ( - Fig. 1). However, due to EMR differences not all sites recorded each of the time variables. Baseline patient demographic data including age, gender, American Society of Anesthesiologists (ASA) score, and procedure classification (inpatient vs. outpatient) were also collected for each case. Study groups were then created based on the location. The primary outcome of this study compared operative times between different medical center categories.

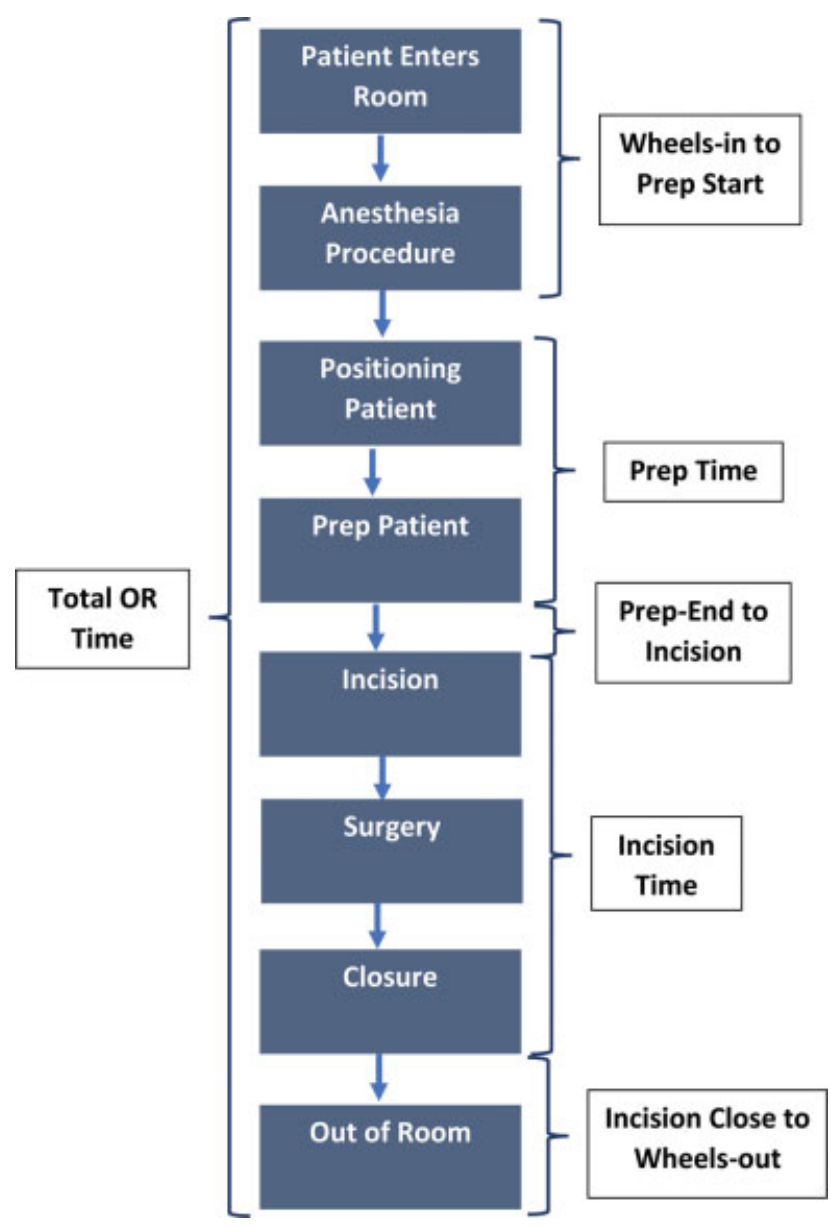

Fig. 1 Operative times.

\section{Hospital Classifications}

Four hospitals were included as part of this study. Each is part of a single healthcare organization in a major metropolitan area. Each hospital was first classified based on the average number of orthopedic cases. High volume was defined as greater than 357 THAs per year, as this has been shown to be a meaningful threshold that yields shorter length of stays and lower complication rates once surpassed. ${ }^{19,20}$ The locations were then separated based on whether they were directly affiliated with the medical school (academic center) and had substantial teaching faculty and research facilities. Location 1 (high-volume academic [HVA]) is a large, highvolume, and academic specialized orthopedic hospital dedicated to inpatient and outpatient orthopedic treatment. HVA was the only site in this study at which only orthopedic cases were performed, and OR staff only took part in orthopedic surgeries. Location 2 (low-volume community [LVC]) is a 450-bed, low-volume, and community teaching hospital, where some HVA surgeons also perform surgery but also includes community-based surgeons. Location 3 (low-volume academic [LVA]) is an academic, tertiary-care hospital, and the flagship hospital of the academic institution. All orthopedic cases done at LVA are performed by the same surgeons as at the HVA institution. However, the cases performed at the LVA site are too medically complex to be done at the HVA location, with significant medical issues 
such as need for dialysis or have high cardiac risk. Location 4 (high-volume community [HVC]) is a 591-bed, larger community hospital with a substantial proportion of orthopedic cases. This particular hospital had traditionally been a community hospital that within the last few years merged with the main academic institution. Each location maintains separate staff and resources despite being under one umbrella organization. As this was a quality improvement project, our study was exempt from institutional review board (IRB) approval.

\section{Surgeon Volume}

Operations at the HVA site were conducted by 60 surgeons with an average yearly volume of $56.38 \pm 78.90$ THA cases per surgeon. Operations at the LVC were conducted by 35 surgeons with an average yearly THA case load of $10.07 \pm 13.06$. The LVA had 32 surgeons performing THA with an average yearly volume of $30.7 \pm 37.27$ cases per surgeon. Finally, the HVC had 10 surgeons who each performed an average of $26 \pm 24.94$ THA operations per year.

\section{Statistical Analysis}

Descriptive data are represented as means \pm standard deviation for continuous data and total counts for categorical data. Chi-square tests were used to find differences in categorical variables. One-way ANOVA analysis was employed to test for significant differences between continuous demographic variables as well as OR times among each institution. Post hoc Tamhane tests were used to determine differences in demographic data and the different OR times between sites. Multivariable logistic regressions were utilized to further compare the OR times to control for patient demographic differences between locations. Findings were reported as $p$-values to indicate a statistical difference between sites. All statistical analyses were performed by using SPSS v25 (IBM Corporation, Armonk, NY). A p-value of less than 0.05 was considered to be statistically significant.

\section{Results}

The primary objective of this study was to determine whether the volume and type of hospital affects THA OR times. A total of 7,694 consecutive patients were included in this study with 7,019 undergoing a primary THA and 675 undergoing a revision THA over a 3-year period between January 2016 and September 2019. Among the different sites, primary THA patient demographic data differed significantly with regard to age $(p<0.0001)$, gender $(p=0.021)$, and ASA class $(p<0.0001)$ (-Table 1$)$. For revision THA cases, only ASA class $(p<0.0001)$ was significantly different between locations (-Table 2). After normalizing surgeon volume data through logarithmic transformation, the HVA and HVC locations had a similar average surgeon THA case volume per year ( 3.25 vs. 2.83 cases, $p=0.718$ ). The LVA and LVC hospitals also had surgeons with similar yearly THA operative volumes ( 2.27 vs. 1.71 cases, $p=0.371)$. However, they were significantly less than the HVA location $(p=0.046$; $p<0.0001$ ). Initial ANOVA analysis revealed that all OR times were significantly different between sites for both primary and revision THA. However, due to significant demographic differences, post hoc regression analysis was required to determine which of these differences existed between locations.

\section{Primary Total Hip Arthroplasty Results}

Among the OR times measured for primary THA, there were few similarities between the sites using regression models. The only two sites with statistically similar times were the two community hospitals (LVC and HVC centers) which had similar total OR times $(175.48 \pm 44.22$ vs. $167.27 \pm 47.19$

Table 1 Patient demographics within the primary total hip arthroplasty group

\begin{tabular}{|c|c|c|c|c|c|}
\hline & $\begin{array}{l}\text { HVA } \\
(n=5,371)\end{array}$ & $\begin{array}{l}\text { LVC } \\
(n=497)\end{array}$ & $\begin{array}{l}\text { LVA } \\
(n=117)\end{array}$ & $\begin{array}{l}\text { HVC } \\
(n=1,034)\end{array}$ & $p$-Value \\
\hline Age & $62.87 \pm 12.23$ & $67.05 \pm 11.54$ & $65.48 \pm 13.85$ & $64.01 \pm 12.11$ & $<0.0001$ \\
\hline Gender & & & & & 0.021 \\
\hline Male & $2,376(44.2 \%)$ & 209 (42.0\%) & 82 (70.1\%) & 490 (47.4\%) & \\
\hline Female & 2,995 (55.8\%) & 288 (58.0\%) & 35 (29.9\%) & 544 (52.6\%) & \\
\hline ASA & & & & & $<0.0001$ \\
\hline 1 & 375 (7.0\%) & 7 (1.5\%) & $0(0 \%)$ & $15(1.4 \%)$ & \\
\hline 2 & 3,489 (65.0\%) & $188(37.8 \%)$ & 30 (25.6\%) & 515 (50.0\%) & \\
\hline 3 & $1,437(26.7 \%)$ & $275(55.3 \%)$ & 76 (65.0\%) & $476(46.0 \%)$ & \\
\hline 4 & 70 (1.3\%) & $27(5.4 \%)$ & $11(9.4 \%)$ & $28(2.6 \%)$ & \\
\hline Patient type & & & & & $<0.0001$ \\
\hline Inpatient & $5,088(94.7 \%)$ & 485 (97.6\%) & 109 (93.2\%) & 1027 (99.3\%) & \\
\hline Outpatient & $283(5.3 \%)$ & $12(2.4 \%)$ & $8(6.8 \%)$ & $7(0.7 \%)$ & \\
\hline
\end{tabular}

Abbreviations: ASA, American Society of Anesthesiologists; HVA, high-volume academic; HVC, high-volume community; LVA, low-volume academic; LVC, low-volume community; THA, total hip arthroplasty. 
130 Shortest Operative Times in Total Hip Arthroplasty Zak et al.

Table 2 Patient demographics within the revision total hip arthroplasty group

\begin{tabular}{|c|c|c|c|c|c|}
\hline & $\begin{array}{l}\text { HVA } \\
(n=523)\end{array}$ & $\begin{array}{l}\text { LVC } \\
(n=34)\end{array}$ & $\begin{array}{l}\text { LVA } \\
(n=29)\end{array}$ & $\begin{array}{l}\text { HVC } \\
(n=89)\end{array}$ & $p$-Value \\
\hline Age & $66.25 \pm 11.35$ & $69.23 \pm 11.86$ & $68.76 \pm 14.09$ & $66.91 \pm 11.58$ & 0.56 \\
\hline Gender & & & & & 0.75 \\
\hline Male & 235 (44.9\%) & $12(35.3 \%)$ & $13(44.8 \%)$ & 40 (44.9\%) & \\
\hline Female & 288 (55.1\%) & $22(64.7 \%)$ & $16(55.2 \%)$ & 49 (55.1\%) & \\
\hline ASA & & & & & $<0.0001$ \\
\hline 1 & 15 (2.9\%) & $0(0 \%)$ & $0(0 \%)$ & $0(0 \%)$ & \\
\hline 2 & 254 (48.6\%) & $14(41.2 \%)$ & $2(6.9 \%)$ & $39(43.8 \%)$ & \\
\hline 3 & 237 (45.3\%) & $15(44.1 \%)$ & 20 (69.0\%) & $47(52.8 \%)$ & \\
\hline 4 & $17(3.2 \%)$ & $5(14.7 \%)$ & $7(24.1 \%)$ & $3(3.4 \%)$ & \\
\hline Patient type & & & & & 0.60 \\
\hline Inpatient & $500(95.6 \%)$ & 31 (91.2\%) & $29(100 \%)$ & 89 (100\%) & \\
\hline Outpatient & 23 (4.4\%) & $3(8.8 \%)$ & $0(0 \%)$ & $0(0 \%)$ & \\
\hline
\end{tabular}

Abbreviations: ASA, American Society of Anesthesiologists; HVA, high-volume academic; HVC, high-volume community; LVA, low-volume academic; LVC, low-volume community; THA, total hip arthroplasty.

minutes; $p=0.578)$. All other times were statistically different between all of the sites. The HVA $(n=5,371)$ specialized orthopedic hospital maintained statistically lower total OR times in comparison to the LVC ( $n=497)$, LVA $(n=117)$, and HVC $(n=1,034)$ sites $(155.96 \pm 38.96$ vs. $175.48 \pm 44.22$ vs. $184.42 \pm 184.28$ vs. $167.27 \pm 47.19$ minutes; $p<0.0001$; - Table 3). Total incision times were also significantly lower at the HVA location in comparison to the LVC, LVA, and HVC locations $\quad(97.32 \pm 32.49 \quad$ vs. $117.32 \pm 37.86 \quad$ vs. $118.08 \pm 19.85$ vs. $110.62 \pm 34.58$ minutes; $p<0.0001$; -Table 4). "Wheels-in to prep start" and "prep-end to incision" were also significantly lower at the HVA location (-Table 5). However, the "prep time" and the "incision close to wheels-out" were slightly higher at HVA than those of the LVC site ( $21.76 \pm 11.26$ vs. $4.02 \pm 2.37$ minutes, $p<0.0001$; $10.07 \pm 6.00$ vs. $8.01 \pm 5.85$ minutes, $p<0.0001)$. It should be noted that this large difference in prep times may be due to differences in recording prep times between sites.

\section{Revision Total Hip Arthroplasty Results}

OR times for revision THA significantly differed between sites with the initial ANOVA analysis. HVA $(n=523)$ and LVA $(n=29)$ had statistically similar incision times (153.02 \pm 61.03 vs. $164.10 \pm 88.23$ minutes; $p=0.22$ ). However, when accounting for demographic differences LVC $(n=34)$ and HVC $(n=89)$ were found to have differing in OR times (194.85 \pm 71.51 vs. $279.54 \pm 76.55$ minutes; $p<0.001)$ and incision times $(132.24 \pm 60.07$ vs. $211.22 \pm 75.62$ minutes; $p<0.001)$. The LVC location thus had the significantly lowest OR times, followed by the HVA, LVA, and finally HVC sites $(194.85 \pm 71.51$ vs. $224.69 \pm 90.57$ vs. $244.07 \pm 66.55$ vs. $279.54 \pm 76.55$ minutes). Interestingly, HVA and LVC had similar incision close to wheels out time $(16.15 \pm 10.30$ vs. $10.62 \pm 7.19$ minutes; $p=0.305$ ) indicating patients were moved from the OR to the recovery area in a similar manner and period of time. The operative times of wheels-in to prep start, prep

Table 3 Multivariate regression analysis comparing of total operative time in Primary THA for each of the hospital setting with $p$-values and unstandardized $\beta$ values displayed in the box corresponding to the two settings compared

\begin{tabular}{|c|c|c|c|c|c|c|}
\hline \multicolumn{7}{|c|}{ Comparison of total operative time between settings for primary THA } \\
\hline & \multicolumn{2}{|l|}{ LVA (184 $\pm 24 \mathrm{~min})$} & \multicolumn{2}{|l|}{ HVC (167 $\pm 47 \mathrm{~min})$} & \multicolumn{2}{|l|}{ LVC (175 $\pm 44 \mathrm{~min})$} \\
\hline & Unstandardized $\beta$ & $p$-Value & Unstandardized $\beta$ & $p$-Value & Unstandardized $\beta$ & p-Value \\
\hline HVA $(156 \pm 39 \mathrm{~min})$ & 18.86 & $<0.0001$ & 3.21 & $<0.0001$ & 19.411 & $<0.0001$ \\
\hline LVA $(184 \pm 24 \mathrm{~min})$ & & & -7.94 & 0.002 & 22.22 & $<0.0001$ \\
\hline HVC $(167 \pm 47 \mathrm{~min})$ & & & & & 0.535 & 0.578 \\
\hline
\end{tabular}

Abbreviations: ASA, American Society of Anesthesiologists; HVA, high-volume academic; HVC, high-volume community; LVA, low-volume academic; LVC, low-volume community; THA, total hip arthroplasty.

Note: Regression analysis included the additional variables of age, gender, ASA, and patient type (inpatient vs. outpatient). 
Table 4 Multivariate regression analysis comparing incision time in primary THA for each of the hospital setting with $p$-values and unstandardized $\beta$ values displayed in the box corresponding to the two settings compared

Comparison of surgical time between settings for primary THA

\begin{tabular}{|l|l|l|l|l|l|l|}
\hline & \multicolumn{2}{|l|}{ LVA (118 $\pm \mathbf{2 0} \mathrm{min})$} & \multicolumn{2}{l|}{ HVC (111 $\pm \mathbf{3 4} \mathrm{min})$} & \multicolumn{2}{l|}{ LVC (117 $\pm \mathbf{3 8} \mathrm{min})$} \\
\cline { 2 - 7 } & Unstandardized $\beta$ & $p$-Value & Unstandardized $\beta$ & $p$-Value & Unstandardized $\beta$ & $p$-Value \\
\hline HVA (97 $\pm 32 \mathrm{~min})$ & 14.46 & $<0.0001$ & 3.20 & $<0.0001$ & 20.98 & $<0.0001$ \\
\hline LVA (118 $\pm 20 \mathrm{~min})$ & & & -4.79 & 0.029 & 11.41 & $<0.0001$ \\
\hline HVC (111 $\pm 34 \mathrm{~min})$ & & & & & -0.493 & 0.54 \\
\hline
\end{tabular}

Abbreviations: ASA, American Society of Anesthesiologists; HVA, high-volume academic; HVC, high-volume community; LVA, low-volume academic; LVC, low-volume community; THA, total hip arthroplasty.

Note: Regression analysis included the additional variables of age, gender, ASA, and patient type (inpatient vs. outpatient).

Table 5 Differences in operating room times and the breakdown of time between the four institutions

\begin{tabular}{|l|l|l|l|l|l|}
\hline \multicolumn{7}{|l|}{ Operating room time comparisons (min) } & HVA & LVC & HVC & ANOVA $p$-Value \\
\hline & $155.96 \pm 38.96$ & $175.48 \pm 44.22$ & $184.42 \pm 24.28$ & $167.27 \pm 47.19$ & $<0.0001$ \\
\hline Primary THA & $17.94 \pm 9.42$ & $34.20 \pm 12.79$ & $22.72 \pm 11.65$ & & $<0.0001^{\text {a }}$ \\
\hline Total OR time & $21.76 \pm 11.26$ & $4.02 \pm 2.37$ & $26.75 \pm 14.19$ & & $<0.0001^{\text {a }}$ \\
\hline Wheels-in to prep start & $8.63 \pm 7.92$ & $12.25 \pm 6.48$ & $6.57 \pm 5.40$ & & $<0.0001^{\text {a }}$ \\
\hline Prep time & $97.32 \pm 32.49$ & $117.32 \pm 37.86$ & $118.08 \pm 19.85$ & $110.62 \pm 34.58$ & $<0.0001^{\text {a }}$ \\
\hline Prep-end to incision & $10.07 \pm 6.00$ & $8.01 \pm 5.85$ & $13.67 \pm 8.68$ & & $<0.0001^{\text {a }}$ \\
\hline Incision time & $224.69 \pm 66.55$ & $194.85 \pm 71.51$ & $244.07 \pm 90.57$ & $279.54 \pm 76.55$ & $<0.0001$ \\
\hline Incision close to wheels-out & $22.9 \pm 12.56$ & $36.65 \pm 13.56$ & $28.03 \pm 15.99$ & & $<0.0001^{\text {a }}$ \\
\hline Revision THA & $25.05 \pm 14.27$ & $3.65 \pm 1.49$ & $30.1 \pm 18.14$ & & $<0.0001^{\text {a }}$ \\
\hline Total or time & $7.56 \pm 7.68$ & $11.71 \pm 4.94$ & $6.14 \pm 4.67$ & & 0.004 \\
\hline Wheels-in to prep start & $153.02 \pm 61.03$ & $132.24 \pm 60.07$ & $164.1 \pm 88.23$ & $211.22 \pm 75.62$ & $<0.0001$ \\
\hline Prep time & $16.15 \pm 10.30$ & $10.62 \pm 7.19$ & $15.69 \pm 9.87$ & & 0.009 \\
\hline Prep-end to incision & Incision time &
\end{tabular}

Abbreviations: ANOVA, analysis of variance; HVA, high-volume academic; HVC, high-volume community; LVA, low-volume academic; LVC, lowvolume community; OR, operating room; THA, total hip arthroplasty.

aDenotes that a regression analysis accounting for demographic differences yielded a significant difference of $p<0.0001$ for all groups.

Note: HVC did not collect all breakdown data.

time, and prep-end to incision were found to be different between all sites using the regression analyses.

\section{Discussion}

As the demand for THA rapidly rises and is projected to grow to approximately $170 \%$ by 2030 in the United States, the need for efficient, cost-saving measures in orthopedic practices to accommodate a larger number of patients, such as decreasing operative times, has become increasingly important. ${ }^{19,20}$ One proposed solution to improve surgical times is to relocate TJA procedures to higher volume centers. Traditionally, larger academic hospitals were thought to have longer operative times due to more complex cases and the time spent educating residents and fellows. However, others have hypothesized that the higher volume of these tertiary academic hospitals leads to more efficient staff and shorter operative times. While a recent study has shown that a pseudo-specialized community hospital in a single health system had shorter operative times for total knee arthroplasty (TKA), we sought to determine if these findings were translatable to THA performed at our institution. ${ }^{15}$

In the present study, we examined the differences in surgical times for primary and revision THA between four medical centers of varying operative volume. For primary THA, the HVA center had statistically lower total OR and incision times in comparison to all of the other sites included in this study, most notably the HVC and LVC sites. These data suggest that our community hospitals are slower than our HVA center which specializes in orthopedic procedures. Interestingly, our LVA center had statistically greater OR times across the board despite utilizing the same surgeons as the HVA center. This could be in part due to the fact that surgeries normally conducted at the LVA site are reserved for 
higher risk patients and not appropriate for a specialty hospital that has fewer medical co-management capabilities such as cardiac intensive care and dialysis. Additionally, the smaller sample size of the LVA location has the potential to bias our results. We found that the incision times at the HVA site were comparable to other tertiary, urban teaching hospitals, whose primary THA times range from 92 to 97 minutes. ${ }^{21}$ While a study by Keswani et al only examined incision times and not total OR times, their findings corroborate ours, suggesting that academic hospitals despite volume, may maintain similar operative times. The claim that similar types of hospitals, regardless of volume have similar total OR times is further supported by our findings which showed that our community hospitals, HVC, and LVC, had statistically similar operative times. This suggests that operative volume does not solely affect operative times but there may be additional factors such as available resources present at specialty hospitals and not community hospitals. A study of 713,573 surgical cases in Canada found that operations performed at academic teaching hospitals often took significantly longer than community hospitals with differences ranging from 5 to 62 minutes. In the study by Dy et al, THAs conducted at teaching hospitals took on average 5 minutes longer. ${ }^{22}$ Conversely, our HVA center had an average total OR time that was 11.31 minutes shorter than our HVC center, and an incision time that was 13.3 minutes shorter. Our results thus suggest that the increased time dedicated to educating residents and fellows about THA in the operating room is offset by greater access to resources such as specialized surgical instruments, equipment, and implants, and specialized staff more familiar with TJA in comparison to community locations. In comparison to the community sites in this study, the HVA hospital had specialized, dedicated orthopedic surgery operative staff, with greater access to implants inventory and instrument trays as well as more orthopedic specific intraoperative technology (i.e., robotics and navigation).

Unlike the primary THA cohort, our revision THA group was much smaller. As expected, revision THA are more complex than their primary counterparts and thus took an overall longer time to perform across all sites. With a limited sample size and no data on the complexity of the revision cases, it is difficult to draw conclusions from our findings. Interestingly, however, the LVC site had the shortest overall OR and incisions times followed by the HVA and LVA sites. The LVC hospital had a small sample size and had a low average OR time. However, this may be due to the fact that revisions, especially complex ones are less likely to occur at community and lower volume hospitals. A study by Dy et al identified over 17,000 revision TJA patients and found that $30 \%$ of patients changed hospitals from their index procedure. $^{23}$ They additionally found that patients who underwent their index TJA at a lower volume or community hospital were more likely to change institutions than patients who had an index procedure at a higher volume center. Notably, these same patients were also more likely to change to a higher volume center. This therefore helps to explain why the HVA center had a larger number of cases than the community hospitals. As expected, the HVA hospital had statistically lower OR times than the HVC location which suggests that specialized academic hospitals may be more equipped to deal with the complexity of revision THA.

We believe that our HVA center was able to maintain the greatest efficiency within our health system since it is an orthopedic specialty hospital. We found that hospital volume is not the primary factor affecting operative times but propose that the staff and resources available at the site are most contributory to these results. With specialized staff and resources for TJA, there is improved continuity of care that can be provided as OR nurses and technicians are able to smoothly transition between cases. We believe our findings show that having an orthopedic specialty hospital/teams allows for more efficient and consistent care for both primary and revision THA. Further studies will be needed to identify the long-term outcomes and cost benefits to the healthcare system for cases performed at different types of medical centers. However, proper training and dedicated orthopedic nursing teams combined with standardized OR protocols and specialized resources found at specialized hospitals can aid in the development of more efficient care for arthroplasty patients.

Not only are these findings important in reducing operative costs, but are also equally significant considering that shorter operative times lead to improved outcomes following TJA. Increased TJA operative times are associated with increased length of stay and increased risk of readmissions, wound complications, and infection. ${ }^{7-10,24-26}$ Longer procedures also have the potential for increased blood loss, hematoma formation, or venous thromboembolism. ${ }^{12,27}$ Additionally, it has been shown that for primary THA, longer operative times place patients at a greater risk for revision surgery. ${ }^{10}$ While the definition of "high volume" varies among studies, many have found that higher volume hospitals, which we found to have shorter operative times, had better outcomes following TJA than lower volume centers. ${ }^{16-18}$ As our study found that our HVA hospital has significantly lower primary THA operative times than our community hospitals, it follows that patients undergoing a primary THA at these hospitals may also experience improved outcomes and is an area for future investigations.

There are several limitations to this study. As retrospective study, our results may be susceptible to selection bias. We attempted to minimize these limitations by only including the data of a consecutive patient cohort over a time period of 3 years. Additionally, since multiple hospitals were included in this study, variation in how each institution records the subsections of their OR times could have skewed the time values. Furthermore, the small number of revision THA cases at lower volume centers makes drawing conclusions more difficult. Data on the specific types of revisions were not extracted for this study, so the wide variation in operative time may also reflect the complexity of revisions performed. Additionally, our results show that hospital volume and average yearly surgeon case volume increase similarly. In the current literature, there is no evidence that surgeon volume affects THA operative times but rather 
surgeons with more experienced have shorter operative times. ${ }^{28}$ However, there is much evidence showing that increased hospital and surgeon volume both reduce postoperative complications suggesting that both have similar affects. $^{29}$ Moreover, our results may not be generalizable to other institutions as our HVA center is a specialized orthopedic hospital. For hospital systems without an orthopedic hospital, community hospitals may in fact be more efficient than the academic hospital such as seen in the study by Khanuja et al, where they function as a pseudo-specialty hospital. ${ }^{15}$ Perhaps more important than the surgery location, the creation of specialized orthopedic teams who have familiarity with implants, equipment, and arthroplasty protocols allow for more efficient operative times.

\section{Conclusion}

In our health system located in a major metropolitan area, the HVA orthopedic specialty hospital had the shortest total OR time for primary THA, which differs from the findings from other recent studies. We believe that this is in part because our orthopedic specialty hospital has dedicated staff who are knowledgeable in procedures being performed, as well as additional resources which allows for even complex cases to be performed in an efficient manner. As more emphasis is placed on value-based care in an evolving system of bundled payments, we recommend that healthcare systems consider performing primary and revision THA at orthopedic specialty hospitals or hospitals with established arthroplasty protocols to efficiently drive down operative costs and improve patient outcomes.

\section{Funding \\ None. \\ Conflict of Interest \\ None declared.}

\section{Resources}

1 Desmeules F, Dionne CE, Belzile ÉL, Bourbonnais R, Champagne F, Frémont P. Determinants of pain, functional limitations and health-related quality of life six months after total knee arthroplasty: results from a prospective cohort study. BMC Sports Sci Med Rehabil 2013;5:2

2 Gandhi R, Dhotar H, Razak F, Tso P, Davey JR, Mahomed NN. Predicting the longer term outcomes of total knee arthroplasty. Knee 2010;17(01):15-18

3 Jenkins PJ, Clement ND, Hamilton DF, Gaston P, Patton JT, Howie CR. Predicting the cost-effectiveness of total hip and knee replacement: a health economic analysis. Bone Joint J 2013;95-B (01):115-121

4 Elmallah RDK, Cherian JJ, Robinson K, Harwin SF, Mont MA. The effect of comorbidities on outcomes following total knee arthroplasty. J Knee Surg 2015;28(05):411-416

5 Singh JA, Lewallen DG. Depression in primary TKA and higher medical comorbidities in revision TKA are associated with suboptimal subjective improvement in knee function. BMC Musculoskelet Disord 2014;15:127

6 Cheng H, Chen BPH, Soleas IM, Ferko NC, Cameron CG, Hinoul P. Prolonged operative duration increases risk of surgical site infections: a systematic review. Surg Infect (Larchmt) 2017;18 (06):722-735

7 Anis HK, Sodhi N, Klika AK, et al. Is operative time a predictor for post-operative infection in primary total knee arthroplasty? J Arthroplasty 2019;34(7S):S331-S336

8 Duchman KR, Pugely AJ, Martin CT, Gao Y, Bedard NA, Callaghan JJ. Operative time affects short-term complications in total joint arthroplasty. J Arthroplasty 2017;32(04):1285-1291

9 George J, Mahmood B, Sultan AA, et al. How fast should a total knee arthroplasty be performed? An analysis of 140,199 surgeries. J Arthroplasty 2018;33(08):2616-2622

10 Ong KL, Lau E, Manley M, Kurtz SM. Effect of procedure duration on total hip arthroplasty and total knee arthroplasty survivorship in the United States Medicare population. J Arthroplasty 2008;23 (06, Suppl 1):127-132

11 Young SW, Mutu-Grigg J, Frampton CM, Cullen J. Does speed matter? Revision rates and functional outcomes in TKA in relation to duration of surgery. J Arthroplasty 2014;29(07):1473-1477.e1

12 Jaffer AK, Barsoum WK, Krebs V, Hurbanek JG, Morra N, Brotman DJ. Duration of anesthesia and venous thromboembolism after hip and knee arthroplasty. Mayo Clin Proc 2005;80(06):732-738

13 Horlocker TT, Hebl JR, Gali B, et al. Anesthetic, patient, and surgical risk factors for neurologic complications after prolonged total tourniquet time during total knee arthroplasty. Anesth Analg 2006;102(03):950-955

14 Namba RS, Inacio MCS, Paxton EW. Risk factors associated with deep surgical site infections after primary total knee arthroplasty: an analysis of 56,216 knees. J Bone Joint Surg Am 2013; 95(09):775-782

15 Khanuja HS, Solano MA, Sterling RS, Oni JK, Chaudhry YP, Jones LC. Surgeon mean operative times in total knee arthroplasty in a variety of settings in a health system. J Arthroplasty 2019;34(11): 2569-2572

16 Wilson S, Marx RG, Pan TJ, Lyman S. Meaningful thresholds for the volume-outcome relationship in total knee arthroplasty. J Bone Joint Surg Am 2016;98(20):1683-1690

17 Katz JN, Barrett J, Mahomed NN, Baron JA, Wright RJ, Losina E. Association between hospital and surgeon procedure volume and the outcomes of total knee replacement. J Bone Joint Surg Am 2004;86(09):1909-1916

18 Hervey SL, Purves HR, Guller U, Toth AP, Vail TP, Pietrobon R. Provider volume of total knee arthroplasties and patient outcomes in the HCUP-Nationwide Inpatient Sample. J Bone Joint Surg Am 2003;85(09):1775-1783

19 Dargel J, Oppermann J, Brüggemann GP, Eysel P. Dislocation following total hip replacement. Dtsch Arztebl Int 2014;111 (51-52):884-890

20 Sloan M, Premkumar A, Sheth NP. Projected volume of primary total joint arthroplasty in the U.S., 2014 to 2030. J Bone Joint Surg Am 2018;100(17):1455-1460

21 Keswani A, Beck C, Meier KM, Fields A, Bronson MJ, Moucha CS. Day of surgery and surgical start time affect hospital length of stay after total hip arthroplasty. J Arthroplasty 2016;31(11): 2426-2431

22 Vinden C, Malthaner R, McGee J, et al. Teaching surgery takes time: the impact of surgical education on time in the operating room. Can J Surg 2016;59(02):87-92

23 Dy CJ, Bozic KJ, Padgett DE, Pan TJ, Marx RG, Lyman S. Is changing hospitals for revision total joint arthroplasty associated with more complications? Clin Orthop Relat Res 2014;472(07): 2006-2015

24 Garbarino LJ, Gold PA, Sodhi N, et al. The effect of operative time on in-hospital length of stay in revision total knee arthroplasty. Ann Transl Med 2019;7(04):66

25 Keswani A, Lovy AJ, Robinson J, Levy R, Chen D, Moucha CS. Risk factors predict increased length of stay and readmission rates in revision joint arthroplasty. J Arthroplasty 2016;31(03): 603-608 
134 Shortest Operative Times in Total Hip Arthroplasty Zak et al.

26 Sodhi N, Patel YH, George J, et al. Operative time, length of stay, short-term readmission, and complications after hinged primary total knee arthroplasty: a propensity score matched analysis. J Knee Surg 2018;31(10):940-945

27 Jibodh SR, Gurkan I, Wenz JF. In-hospital outcome and resource use in hip arthroplasty: influence of body mass. Orthopedics 2004;27(06):594-601
28 Yasunaga H, Tsuchiya K, Matsuyama Y, Ohe K. High-volume surgeons in regard to reductions in operating time, blood loss, and postoperative complications for total hip arthroplasty. J Orthop Sci 2009;14(01):3-9

29 Koltsov JCB, Marx RG, Bachner E, McLawhorn AS, Lyman S. Riskbased hospital and surgeon-volume categories for total hip arthroplasty. J Bone Joint Surg Am 2018;100(14):1203-1208 